\title{
Macro Biophysical Physiological Neuropsychotherapy: Theory and Practice
}

\section{Yakov $\mathbf{N}^{*}$}

National Israeli Center for Psychosocial Support of Survivors of the Holocaust and the Second Generation, Netanya Branch, Israel

\begin{abstract}
Neuropsychotherapy is a macro biophysical, physiological method to therapy that sharpens awareness inpatients with transient homeostatic deregulated (THD) lasting distress of relapse, which produces body operational ranges (BOR) with morbid symptoms, against which a neuropsychotherapist had designed specific sets of tools and skills to train for more effective control over BOR rather than with drugs to eliminate THD, leading to a transient homeostatic resynchronizing (THR) state of remission with a tranquil body that produces pleasant feelings of health The author of the article performed a breakthrough in neuropsychotherapy with special tools and strategies that were built during the acquisition of a placebo effect during self-navigation of the macro biophysical physiological operations through treatment and prevention. It focuses on training patients to develop well-targeted self-awareness to block all stress-inducing sources. The latter originate and sustain macro biophysical physiological distresses leading to transient homeostatic deregulated (THD) relapses, driving uncoordinated body operational ranges (BOR) with morbid symptoms. In neuropsychotherapy the self-intelligence level stimulates learning to positively strengthen the emotional levels monitoring quantify stages in the body operational ranges (BOR) for healthy activities. Neuropsychotherapy filters the cognitive, emotional, and behavioral tools that guide BOR with a set of constellation of antecedent conditions (COAC) to be described below:
\end{abstract}

1. Using 'positive connotations' for developing robust insight,

2. Driving personal BOR into a restful and peaceful state of mind,

3. Providing useful concrete anti-stress strategies and techniques, Using 'here and now' empathic, dynamic, 'doctor-patient alliance',

4. Using personal assertive interactional and conversational skills,

5. Using personal problem-solving tasks, and

6. Setting up family and social COACs. Current neuropsychotherapy is the cutting edge over all other psychotherapies.

Keywords: BOR; THR; THD; COAC; Biophysical Physiological Mind

\section{Introduction}

The central point here is that traditional psychotherapy is replaced by focusing on physiological instructions, means and tools counteracting or preventing physiological ('functional') distresses [1,2]. It indicates that macro biophysical physiological neuro-psychotherapy favorably influences, treats and prevents physiological distress-inducing relapses. Given that the typical current technological society overwhelmingly places dominant competitive burden upon personal over sensitivity to stress factors, require adequate type of monitoring physiological distresses. At baseline, psychopharmacology, brain electrotherapy and other brain-biophysical mindtherapies no common denominator with neuro-psychotherapy $[3,4]$.

Another cardinal point is that neuro-psychotherapy addresses the mission of reframing personal etiological stress-inducing factors imbedded within their external harmful constellation of antecedent conditions (COAC). In this respect, chronic COAC inflicts personal physiological distress levels. What makes neuro-psychotherapy most attractive? It is the fact of having ability to weaken core pathophysiological elements of many 'functional' neuropsychiatric disorders. While there are multiple life event scenarios bearing multiple personal stress-inducing factors in any space encounter, neuronal webs connectivity loops suffer transitory 'functional', microstructural blockages and shunts across rows of ion channels, shifting true biophysical information units into fault cerebral working memory centers. Apparently, neuropsychologists must always keep in mind that clinical evidences show that 'functional' Neuro-pathophysiology fluctuates between relapses with symptom- inducing and remissions with symptom-free states! With relevant information, it is most likely to unite all 'functional' neuro-pathophysiological conditions under a coin name - transitory homeostatic deregulation (THD). Why? One of the best important steps in Neuro-psychotherapy is the fact that neuropsychologists possess strong awareness about underlying pathophysiological routes generating varieties of clinical manifestations. As a result, this advantage makes them more prone for adopting basic principles for gaining proper tools and skills replacing THD with transitory homeostatic resynchronizing (THR) remission levels sustaining it in long run.

*Corresponding author: Yakov Naisberg M.D, AMCHA - Branch Netanya National Israeli Center for Psychosocial Support of Survivors of the Holocaust and the Second Generation, Netanya, Mendel Zinger St. 13/2, Haifa, Israel, Tel: 048341966; Fax: 04-8341966; E-mail: naisberg@012.net.il

Received December 25, 2017; Accepted February 01, 2018; Published February 10, 2018

Citation: Yakov N (2018) Macro Biophysical Physiological Neuropsychotherapy: Theory and Practice. J Ment Disord Treat 4: 156. doi:10.4172/2471-271X.1000156

Copyright: (c) 2018 Yakov N. This is an open-access article distributed under the terms of the Creative Commons Attribution License, which permits unrestricted use, distribution, and reproduction in any medium, provided the original author and source are credited. 
Like in traditional psychotherapy, Neuro-psychotherapy uses face-to-face interactional dynamics based on 'here and now' stressfree framework encounters between individuals, groups, couples and providing guidance for in-family (social) interactions. The classical point here is that unlike traditional, it uses pro-medical model eliminating or protecting subjects at risk from exposure to social etiological stressphysiological distresses! During such cardinal processes neuronal webs connectivity loops pathogenesis weaken. In other words, it is possible to re-mutate abnormally mutated genes in the long run into less abnormal or maybe normal to be proved in future research inquiry. In meantime, gradually clinical signs and symptoms subside and personal tranquil and wellbeing emotions appear, finalizing THR remission arrival! Explicitly, two basal signs persistently indicate on the presence of THR remission level, tranquility and positive emotional charge! Neuro-psychotherapy reinforces and encourages theses basics, helping gain skills to protect and prevent new relapses. One major obstacle for advancing Neuro-psychotherapy is the lack of capacity to non-invasively screen and objectively measure biophysical physiological factors operating behind relapsed THD and THR remissions emergence. Both, neuropsychologists and patients have a strong incentive for controlling the efficacy of treatment with an objective tool of measurements! In the meantime we must keep operate in hopes that such fundamental need will find its realization in an assembled macro biophysical analyzer, offering objective biomarkers evidencing relapse and remission alike [5-7]. The aim of the given article is two folded. First, eliciting basics of materialistic inseparable body-brain-biophysical mind's interplay in health and pathology. Second, how and why it is crucial presenting such superior macro biophysical physiological neuro-psychotherapy sets of placebo-like effects generating alpha rhythmic brain-mind EEG (electro-encephalographic) waves double controlled by therapistpatients interaction, focusing patient's intelligent level to prophylaxis and treatment of psychiatric (psychological) disorders including synesthesia!

\section{Cortical spreading depression (CSD) blocking ionic information flows via neuronal membrane ion channels, shunting it into non-specialized routes}

The term CSD were given by Aristides Leao [8] who decipher EEG charts attributed to brain's pathophysiology. Other researchers have found a number of brain disorders such as metabolic, vascular and traumatic disorders having micro neuronal changes with regard to CSD [9-12]. It turned out that micro and macro CSD run in periodic cycles around fluctuations from regeneration to deterioration and vice versa [13-16]. To be more accurate, by using these facts of replacing one mode to contrary another, the author of the article argued that laws of physics $(5,6,7)$ help replace THD with THR. This can occur only, when the pathophysiological distress temporarily alters neuronal membrane ionic channels across some brain micro regions, and real information units will bypass this flow of information into non-specific routes of neuronal communication, literally to non-specific working memory centers. There, they generate neuropsychiatric prominent signs and symptoms. As with any change, although physiological distress either accelerates or decelerates body operational ranges (BOR) in stages, leading to functional transitory homeostatic deregulation (THD) in neuropsychiatric disorders getting below an elaboration. In general, neuro-psychotherapy uses one of the fundamental CSD evidences in its theory and practice.

\section{How to understand abnormal physiological symptoms without 'organic' pathology?}

The main point here is that one should make an effort explaining all psychosomatic ('functional') illnesses originally created by the same common denominator. It assumes that all of them face fractional interference with ionic biophysical information flows through blocked neuronal membrane ionic pathways, transporting them into faulty working memory centers deregulating a given body(s) entity, decoding its malfunction in a sort of different psychosomatic symptoms. Similar to vascular thrombosis generating collateral blood circulation, so presumably occur at vulnerable blockages across microscopic sets of neuronal membrane ion channels. Two prominent patterns characterize such events. The first, relates to any biophysical physiological distress more likely associated with chronic personal stressors! The second, relates to accumulation of vast numbers of adjacent local neuronal membrane ion channels blockages generating sound signs and symptoms! As we presumably face their origin, the morbid ionic information flow shunts loop looks like:

${ }^{*}$ Non-discriminant pain center producing chest, back, joint, abdominal, trigeminal, hip, shingle or flares of pain.

*Non-discriminant allergic center originating - itching.

Otherwise, non-specific sensory information may run into the motor (executive) centers generating motor symptoms like:

- Motor center - increased rate (moto agitation or tremor), while decrease rate delayed speech or delayed action in a unit of time.

- Body operational ranges (BOR) center - velocity accelerated peristalsis (diarrhea) or velocity-decelerated peristalsis (constipation).

- Respiratory center - short breath.

- Cardio-vascular center - increased heart rate (tachycardia) or decreased heart rate (brady cardia).

- Diaphragm center deregulation - emergence of belching and hiccups.

A pivotal point is here to underline that excitation and inhibition processes in the brain are under dual control. Synchronic biological clock of circadian cycles tuned with given environmental /social and genetic age-related span factors, driving around 24 hours a day incoming information units into working memory centers in the brain [17-19]. Basic substantive evidences indicates that in either case of over-excitation or over-inhibition there should be a deficit in personal Will to control over concentrated focus in relation to sustenance of its timing span. Under these conditions, automatically deregulated brain controls shifts of incoming new sensory or retrieved messages from memory repositories in fragmental processes. Hence, it will create multi pathological signs of:

- Inattentive or absent-mind type behavior.

- Inability processing and reprocessing integrated messages.

- Inability to sustain normal memory function since fragmental information pieces entering working memory centers with fragmental content of any life event scenario.

- Inability to carry out any personalize productive dual or group interaction.

- High probability in self-failing for lack of gaining new knowledge.

- Inability to overcome self- and others barriers for social, academic and other crucial aspects of integration. 
In this respect, an additional point here is that one must be able to explain uncontrollable thoughts, feelings and actions generated by automatic operations. The explanation is that, voluntary centers undergo blockage of input commands running through fault neuronal routes bearing authentic but, in essence, false information flow creating a double problem. First, inability to identify the individual error, because the 'central censor' is blocked! Second, the message center prepares and executes the task, by accident entering into an automatic malfunctioning neuronal route!

One can understand the medical upheaval that impairs normal growth and development during childhood, adolescence, young adulthood leaving such person behind peer achievements. Furthermore, abnormalities of such ranks produce grave and fundamental inability to function in technological societies, blindfolding both, subjects at risk and their local communities accordingly. Such subjects incorrectly treated may self-depart from social compatible into self-indulged antisocial movements with detrimental consequences. The main point here is that this kind of pathology leads to known hyperkinetic disorder. In contrast, over-inhibition generates retarded types of sensory and motor information processing with multi pathological formation too:

- Inability to synchronize with any dominant out of many external information-emitting sources.

- Inability to monitor self-listening to any vocal source delivering speeches on average or higher speed-related rates.

- Inability to monitor performance of tasks requiring average or higher productivity.

- Inability to monitor body operational ranges (BOR) lacking Will control over metabolic energy production and usage.

- Inability to reach social compatible assignments and labeled as a 'looser, lazy or spoiled'.

Of course, one may refer subjects at risk with this neuropsychopathology to hypokinetic disorders. It should come as no surprise that feeling of physical weakness, debilitation or prostration drive them for medical assistance. However, as in many cases, without noticeable 'organic evidences' such patients get inadequate help. Along those lines, self -and community label such individuals 'lacking selfconfidence,' having an 'insecure personality' holding a 'low self-esteem', 'failing in correcting tasks', manifesting 'self-blame and guilt'. For this reason, it is clear that the stigma is born for medical reasons! Those professionals were wrong in none promoting the theory and practice of macro biophysical physiological neuropsychiatric (neuropsychological) remedy. Such patients, except of suffering, pay for an extra biased social labeling!

\section{Cerebral center deregulating alphabet letters}

The chief point here is that complex words and sentences have a common denominator in any human community. Every object located across any ecological environment contain typical for it flora, fauna, animals and human beings within life event scenarios. All of them received noun names with terms of their structural figures, meanings, representations and how they operate with regard to other events. Each word has a basic sequence of letters that makes up a writing expression, linked with reading and talking sounds. Sequence of letters and words of any sentence must get attached to certain singular rows of neuronal membrane ionic channels that in complex present words. The author gave for such wire-like route a term, neuronal electric pathway (NEP). And, one may assume that at least some neuronal membrane ionic channels transmit information from the periphery to the center and vice versa (feedback) about any event. Since eyes hold technologicallike vision perception, it is most likely that pixel-analog images reach cerebral visual centers recording bit-by-bit any object, subject and life event scenario. All those identifiable entities have been attached to basic sizes, shapes and figures, obtain evolutionary abstract 'wordterm' known in any language. Such 'word-term' interconnects writing, reading and speaking domains in a tuning pattern, acting adequately and properly within homeostatic frames of reference.

Another essential point here is that any configuration of a single letter and their sequence composing a word-term should get a pixellike optical configuration engaging finite numbers of NEP's finalizing its resolution a definite singular image. Such constructions hold true for any external object, subject and life event scenario with geometrical configurations, intertied with visual, vocal, hearing, smelling touching and tasting attributes of a given entity. In case of deregulated NEP's one may omit letters in writing, reading and talking without any self- awareness of mistakes originating attention deficit hyperkinetic disorder (ADHD).

\section{Cerebral center deregulating life event conditions}

The focal point here is that patients must be strongly aware that automatic control of executive centers applied cognitive, emotional orders and send off wrong tasks without self-correction. Moreover, this is because of blocking the 'critical censor'. Let us assume that sensory information or real executive orders may get through shunted routes into instant stress-response inducing feedback. At daytime, the person experiences an excessive 'fight or flight or freeze' of 'internal catastrophic event' or known to clinicians as an acute panic attack. At nighttime, such a person suddenly awakes after a 'nightmare fear', followed by almost the same panic attack. Another scenario would follow the general physiological distress, reshaping homeostatic positive for negative experience, due to changes in the physiological deregulatory makeup. The key point here is that it also builds new neuronal web connectivity loops under activated vulnerable memory repositories with encoded unpleasant life event scenarios. As long as this condition lasts, such person experiences types and levels of depression. She/he suffer overwhelming intrusive thoughts looking like one who is introspectively incessantly self- occupied with 'chewing ruminations'.

\section{Will center regulating attention cursor-like indicator}

The fundamental point here is that metabolic energy in a state of homeostasis mobilizes and uses proper quantities in per any unit of time. Under homeostatic deregulation chaotic changes occur. If, for instance, high percentage of homeostatic metabolic energy production cuts off supply to the Will center, assumingly macro biophysical physiological deregulation would destabilize the overall ongoing processes. Taken into account that parallel information processing occurring across neuronal electrical pathways (NEP's) compete for attracting the attention cursor satisfying full awareness of the content and form of knowledge running in, than low numbers of NEP's supplying metabolic energy may cause lacking ability for discrimination. Assumingly, under such deregulated metabolic energy supply, the Will loses its capacity ruling information competition and arbitrary would react to any kind of sensory information as a leading source for attention focus. Such mixed pathology express patients who suffer from schizophrenia. In case of, their incoming visual information fractionally shunts into auditory working memory centers, they will have verbal hallucinations, adding misjudging external visual scenario as a 'plot against them.' 


\section{How to explain the nature of positive and negative emotional intensities driven by body operational ranges (BOR)?}

The primary point here is that circadian cycles drive daytime homeostatic operations due to external loading demands and genetic control takes place at nighttime sleep phases. At onset of sleep BOR inhibit speed-decelerating stage 4 (traditional accountable for sleep phase 1) through sequence of BOR sleep phase 2, 3 and 4 . After completing sleep phases in the reverse sequence phases accelerate BOR to accomplish sleep. Brain excitatory biophysical physiological function drives conscious wakeful BOR from 5th resting to 6th under minimal, 7th moderate and eights (8th) maximal physical, mental (biophysical physiological) or mixed loadings. In health, this sequence repeats providing in parallel from all over body-brain-biophysical mind systems synchronic biophysical physiological signals. They run upward into limbic center integrating them like positive emotional valence experiences. The higher BOR stage is in, the stronger positive feelings appear. Strikingly, the opposite take place during transitory homeostatic deregulating (THD) conditions. In that case, BOR stages are in the range of above upper homeostatic threshold or beneath lower homeostatic threshold of reference. In this manner, desynchronized biophysical physiological signals arrives into limbic emotional disintegrated centers providing negative emotional valence experiences. One should remember this point, in case while BOR stages operate above upper threshold of homeostasis a person obtained an agitated type of depression, conversely, BOR beneath lower threshold levels drive a person into retarded depression. The bottom line here is that under homeostatic frames of reference a special limbic center receives from overall organismic organs and systems synchronic signals confirming their concerted functions under positive selfexperience. And the main point here is that most likely, that resting wakeful stage always bears 'placebo effects' the basal signals interpreting self-experiencing BOR level (stages 5 , or 6 , or 7 , or 8 ). Because of it, the 'placebo effects' emotional biomarker corresponds with BOR level and, in particular, matches BOR and emotional valence in the same intensity levels. Virtually by definition, both represent two separate physiological entities, becoming inseparable in function by macro biophysical physiological evolutionary mechanisms. They generate a double self-alert biomarker for one's crucial need to know about her/ his internal state in health and pathology. Neuropsychologists must teach patients why it is vital using their personal evaluation mechanism that should help them to apply for differentiating self-states in case of need. One advantage of it is that neuropsychologists should manage an active systematic replacement of THD with THR remission. The other advantage of it is that biochemical unitary elements appear integrated into BOR biophysical velocity-related levels, by correcting BOR; one corrects the underlying biochemical profile too. With the advance of macro biophysical physiological neuro-psychotherapy, it is highly important to pinpoint that relapses and remissions run in fragile courses and in two contrasted conditions. The power and general applicability of neuro-psychotherapy should clearly exploit its macro biophysical physiological operational mechanisms and expertly navigate fragile conditions with replicable strategies and tools to strengthen the process of recovery. Neuro-psychotherapy recommends, if under stress the physiological distress drives ones, let say BOR stage 8 level, practically it is possible to reduce it to stage five [5] at rest and an individual should employ every suitable skill she/he acquired to achieve the resting stage 5 and experience tranquil state with pleasant emotions. As one may face these facts, eventually one should proceed in adhering to personal macro biophysical physiological principles keeping tranquil states with self-experienced positive emotion in the end with a sense of cognitive wellbeing of health. Such mechanism depicts (Figure 1).

\section{MBPNOM De-stigmatize "Mental" \& De-fragmentize Medical Prevention}

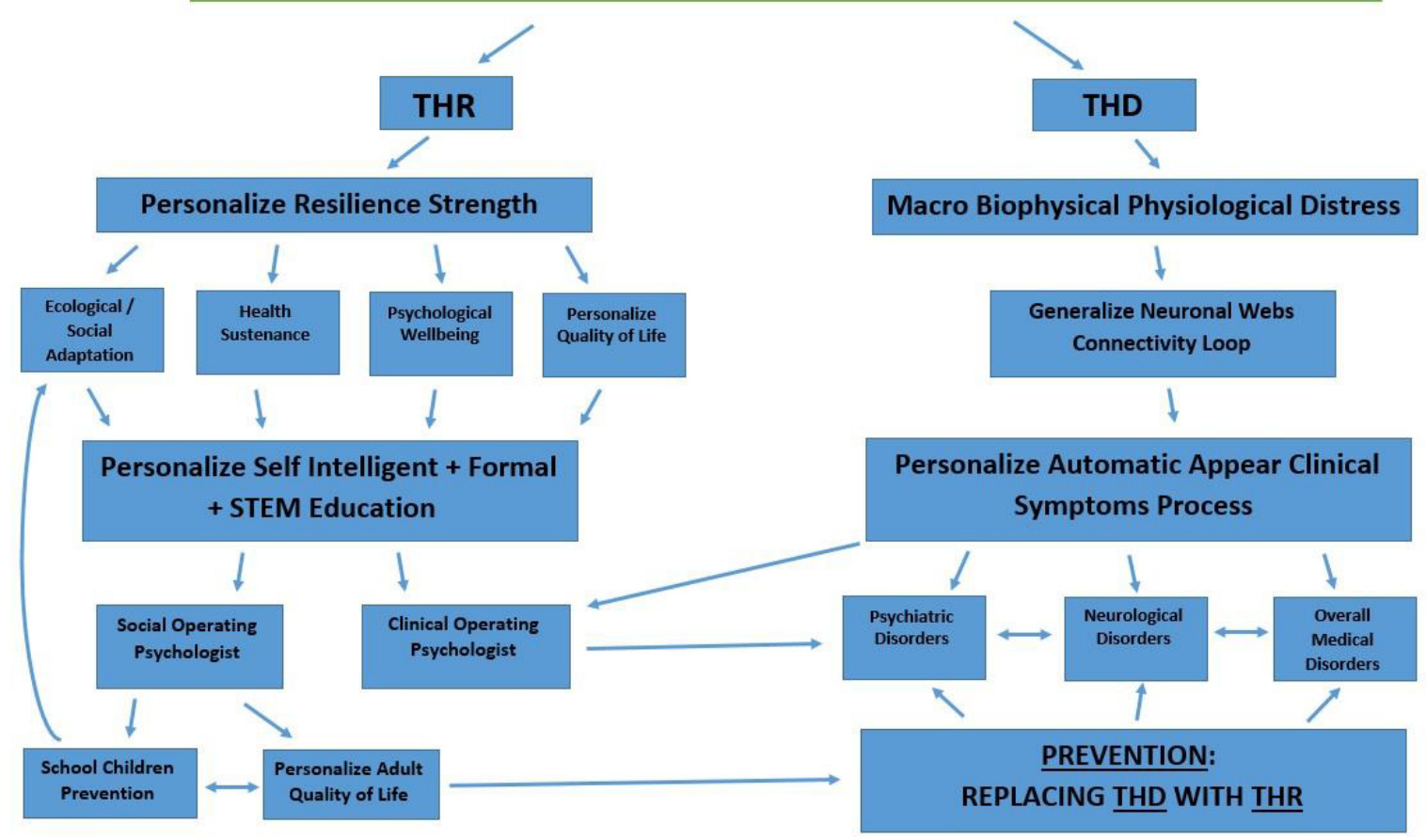

Figure 1: Neuropsychotherapy: The Operational Model. 


\section{Neuro-psychotherapy: Macro biophysical physiological neuropsychiatric operational mechanisms in the origin of THD substituted with THR remission}

Neuro-psychotherapy defines acts that knowingly navigate macro biophysical physiological mechanisms driving placebo effective strategies, tools, means and skills for creating a salient physiological substitute of THD with THR remission. This is the first time in the history of psychotherapy, that neuro-psychotherapy introduces a central focus holding in theory and practice accurate handling destabilized for stabilize physiology. For this to be true, the author of this article anticipates that eventually a macro biophysical analyzer (MBA) will arrive into the practice and along with suited psychological scales shall contribute in obtaining valid and reliable inter-correlated parameters for monitoring its utility. As pinpointed, it highlights clinical advantages that by precise monitoring BOR levels, one should change emotional nature and its intensity. Hence, what makes neuropsychotherapy superior over existing traditional psychotherapy, psychopharmacology, electrotherapy and others in the field of interest? The main method of neuro-psychotherapy aimed in navigating all active therapeutic strategies. That is, using all physical means (exercises, calisthenics and so on), tools and techniques that employ concrete tasks and versatility to replace the unbalanced with physiological balanced levels. The critical point here is that it actively entrains a full set of personal armament and shows the way of navigating self-pace with pinpointed steps that precisely lead to desired outcomes. In this line of practice, one should learn paradigms on essentials for predicting and self-directing systematic favorable leads in

1. Navigating properly BOR with treatment approaches substituting transitory homeostatic deregulation (THD) with transitory homeostatic resynchronize (THR) remission for years.

2. Adequately acquire needed strategies and tools to achieve such results.

3. Becoming aware to self-control own body operational ranges (BOR) under comfortable pace tuning into body tranquility with an inseparable positive emotion. Both biomarkers indicate on the presence of a transitory homeostatic resynchronization (THR) remission!

\section{Personalize Biophysical Resynchronizing Neuro- Psychotherapeutic Principles}

Taken into account that any neuropsychiatrist knows by now fundamentals of neuro-psychotherapy, an expert directly emits verbal and non-verbal physical information messages influence patients' biophysical physiological tranquil tone and positive emotions'. The rational for it lies on protecting patients' dignity, respect and 'always giving positive connotations' to patients behavior' presenting it as a type of 'expression' serving no kind of her/his real desired purposes. In this view, neuro-psychotherapist mobilizes the critical step in securing gain patients cooperation for entraining basic tools and learning paces. After realizing this, one proceeds in entraining interpersonal assertive interactions, anti-stress encounters, and problem solving techniques, creating stress-free COAC and many strategies for healthy daily life event scenarios and good quality of life sustenance. A particular contribution in crystallizing these principles were refined from working shoulder-by-shoulder with clinical psychologists and psychotherapists in our current agency practice. In the presence of an appropriate leadership by the Chief Clinical Psychologist who flexible and expertly leads the team workers practice, a mutual professional learning enrichment occurs. As we respect each other, mutual patients obtained proper medical guidance without contradicting their flexible steps in helping aged Holocaust survivors to go through their upheavals. This favorable sequence of events, prove to be profitable in a spot training without breaking traditional approaches, helping to generate a beneficial professional atmosphere for medical practical acceptance. However, beyond such phenomenon remains the cognitive, emotional and behavior principles directly influencing personal physiological conditions. Consistent with these states, clinical psychologists and psychotherapists who adopt some of neuro-psychotherapy principles actively guide patients BOR driving them into tranquil experiences with inseparable positive emotional intensity. Below there are some examples of neuro-psychotherapy principles anticipating entraining into one's awareness; if one adheres to following statements, she/ he should know that they nurture favorable physiological operations feeding rectifying mechanisms of sustenance by:

- Avoiding raising expectations for high self-achievements in closed communities. It may lead to stress-inducing events from any disappointment acting like stress.

- Categorically, avoiding taking irresponsible critical steps in solving vital solutions, while one feels distressed or anxious. One should keep away in hurry taking critical steps; one should take her/his own time to reason it over!

- Keeping to mutual decisions and avoiding making unilateral steps regarding mutual interests. They upset mutuality and put one on linear lasting physiological distress.

- Considering self-regulating private issues with good judgement due to local social common way of life. Never get into anticipate stress-inducing situations.

- Self-teaching adjusting to compatible cooperation, otherwise competing is stressful.

- Always sticking to accomplish tasks and jobs at time. Do not avoid them and protect self from stress-inducing biased labeling.

- Avoiding looking for absolute justice, it has to do with employing your wisdom. In proving justice, you pave the road to many stress factors.

- Avoiding getting into unnecessary debates or quarrels. One may win the argument, but in the process losing her/his own health. It is a heavy price to pay!

- Learning listening helps understanding other people's intentions. It is better to listen without interruptions, rather than give some endless speeches under stress.

- Treating carefully one's verbal expressions and never trying blackening others. It may come upon one as a boomerang with grave stress.

- Avoiding prejudging oneself heavily. One does not deserve self-labelling and a low self-esteem. One does not need neither self-blame nor self-shame!

- Avoiding discussing one's intrusive thoughts or emotions either with oneself or with other people. This merely protects one from strengthening bonds in one's physiological deregulation.

- Avoiding imposing one's Will upon others, they may reject with using unbearable stress- inducing factors. 
- Avoiding using terrible interpretations about other people. One should know that negative views of others, might turn back on with many stress factors.

- Avoiding dominate friends, one should take an equal standing. Domination attracts many unpredictable stress factors.

- Avoiding loss of 'internal brakes', clearly knowing one's vulnerable points and learning prevent instant outbursts of anger responses. One must learn how to regain control in every stress-inducing life event scenario!

- Knowing that one is oversensitive and susceptible to stress, even to ordinary life event scenarios. One should acquire solid tools and skills helping to protect oneself!

- Knowing that having a strong self-incentive should help one in 'here and now' situations. Always just use stress-free encounters guaranteeing one better quality of life!

- Being courteous to everyone may protect one from others intentionally sets of stress factors.

- Acknowledging that there is no replacement for life, biophysical physiological health, psycho (macro biophysical physiological) wellbeing and personal good quality of life, substantially shall protect one from psychological distressful life events.

Subjects at risk should not repeat most troubled self-inducing stressful statements, because, in fact, they generate rehearsals to strengthen ties with links in abnormal neuronal web connectivity loops. As long as it may take, such subjects at risk should comprehend, that their improper self-inducing hurry for solving problems drives their consideration into a stress-inducing paths with biophysical physiological distress influencing their mutated vulnerable genes leading to vicious circles.

In fact, one should learn from self-experience truly interpreting physiological sensations emitted and felt by anyone under laborious physical or biophysical (mental) tasks. Therefore, fundamental knowledge about underlying physiology that is beyond classical Neuro psychotherapy is the best truthful source on which experts and patients should rely and masterly protect anyone from sliding into 'eternal physiological distressful suffering'. There is a bunch of hope that neuro-psychotherapy along with side effect-free medication, balanced nutrition, balanced sleep sustenance, balanced physical exercises within daily scheduled life framework of occupation, recreation may replace macro biophysical physiological distresses with THR remissions. Deep understanding of advantages that offer this kind of neuropsychotherapy for any practicing psychotherapists, may add to patients advantage in the long-years of such sustained psychotherapy. One may anticipate re-mutation of former abnormal genes into normally effective genes shall take to be proven in future research.

\section{Universal Guidelines for Neuro Psychotherapy}

Before giving clinical examples, the author adopted an intervention method based on systematic and uniform clinical orientation, directing each individual case to receive the same guiding points design to shorten intervals of any relapse and reduce intensive levels of morbid symptoms, until they disappear and a new remission intensifies.

First, the neuropsychological biophysical physiological approach directs the expert to attract the attention of the patient to a keen listening to his 'positive interpretation' of the negative behaviors adopted by the patients' stress-induced view of their immediate surroundings, such as feelings of disrespect, disregard, lack of value, insecurity, inability, deprivation, biased prejudgment and so forth.

\section{Rationale to give a 'positive connotation'}

Patients who enter the treatment have high expectations for each therapist. They bear prolonged negative experiences towards previous caregivers who created a negative feeling and opinion about their own approach to them. These experience hard loads of disappointments and lack of confidence before entering therapy. In order to gain their cooperation at a first meeting, it is essential for neuropsychologists or psychotherapists to emphasize that the patients are not to blame for their negative reactions and behavior towards their immediate environment. In the absence of prior knowledge, some of them used, for example, drugs to feel fun, others use excessive running for pleasant excitement, and others break out due to impulsiveness, or "internal brake loss" and after stress diffusion felt better. They receive a tailored message: 'You are not to blame because you did not know that a healthy body automatically provides a sense of pleasure or fun. Help me to help you please'.

Second, this method primarily strongly motivates the patients for repeating tasks in order to develop body tranquility under pleasant emotions through exercises, blocking intrusive thoughts, shifting attention to scenarios of neutral or positive life events, walking on the beaches, listening to beautiful music, reading attractive brochures, or watching love movies or sport games may replace transitory body operational ranges (BOR) of the transitory homeostatic deregulated (THD) state into a transitory homeostatic (THR) remission.

\section{Rationale for replacing negative emotions with positive emotions}

emotions motivate human forces to lead themselves in the search for every opportunity to experience pleasurable inner feelings. In the absence of current knowledge, many professionals and patients have not known that the healthy body operational ranges (BOR) states of the inseparable body, brain and mind send biophysical signals to the limbic emotional center of the brain that re-processes them into pleasant emotions. Therefore, a healthy body provides a gift for a healthy person with pleasant feelings. On the contrary, for example, addicts extract such feelings from improper substances or medium. Now it is possible to use the method of preventing addiction with training children in school, young people, adults and the elderly to maintain healthy pleasure. Third, neuropsychologists apply the prerequisites required to guide patients to "here and now to pleasant experiences," because these are so vital to the encouragement of self-monitoring tools and means to be properly guided by neuropsychiatric empathic training to help revitalize scenarios of daily dynamic life events with the stages of BOR that produce pleasant feelings. Patients are encouraged by the use of positive (balanced) life events scenarios in physiology and thus stored in such memory storage. The positive physiology succeeds in slowing down the speeds of the negative BOR, which is guided by a state of transient homeostatic deregulation (THD), thus the biophysical and physiological background slowly reverses the balance by achieving a condition in normal transient homeostatic regulation (THR).

\section{Rationale for directing patients on positive 'here and now' life events, replacing negative ones}

Most chronically ill patients bring negative experiences to their professionals. Most of them, even in family conversations or social gatherings, discuss this. This approach stimulates neuronal webs connectivity that transmit negative information in increased 
physiological activity in BOR that will be injected into the brain and will translate into negative emotional feelings that maintain high levels of THD. All of this teaches negative feelings boosted by discussing stress-inducing contents or by self-experience of intrusive that produce bouncing in a negative macro biophysical physiological operations to endanger the body in the patient with the appearance of additional morbidity. The new neuro-psychotherapy is used to reshape negative experiences that may cause impulsivity (internal brake losses) as a result of blocking voluntary regulation, and the brain and body will be activated by automatic impulses. Treatment of guidance in the sense of practicing positive life events in the present gives a tremendous advantage. Because only in this dimension there is complete control over the blockages of stressors and life events can be pre-emptively pleaded in a positive manner. It should be noted that when positive experiences are achieved, they cross a negative physical background, thus weakening negative forces until they are eliminated and the memory of a painful event in the past will receive a neutral loop that will allow such a person to function properly. It is also essential to know not to deal with a predetermined judgment of future life events and just concentrate on the here and now. Expression: 'Cross the bridge when we get there' encourages patients not to waste a positive experience on accurately predicting future life events, because even this might lead to unnecessary stress. Fourth, personal raining of patients to acquire tools, means and strategies against stress.

\section{Fourth, personal training of patients to acquire tools, means and strategies against stress}

In order to overcome the ongoing stressful life event scenarios, a person must be clearly aware of the deep meaning of own macro biophysical, physiological negative feelings that occur after a real incident or imaginary event (retrieved from memory storage recorded past life event). Chronic patients developed a heightened sensitivity to all life events without any differences in negative intensity, thus stabilizing, establishing, and bullying them with impulsive reactions. Patients must undergo learning and re-awakening to a strong selfawareness about their over-sensitive causes and how to weaken them to the ground. They must rely on identifying signs of contributing factors such as disrespect, lack of value, low self-confidence, low self-esteem, low self-efficacy, a sense of persecution, and so on. All of these help to put the training in top order to ensure that she / he has practiced the techniques that work well against personal stress in the specific situation without the need to prevent essential encounters.

\section{Rationale for training personal anti stress tools, means and strategies}

It is not surprising that particular consideration should be given to the understanding of stress processes in the whole body following the exposure to the source of stress, which produces a strong distress across inseparable macro biophysical physiological body, brain and mind operations. The emphasis on this sharpens the awareness of the individual and requires him to listen to professional explanations in order to spur himself and demonstrate seriousness in the process of acquiring the most appropriate tools. In particular, with tools, mediums, and strategies, patients must spend most of their time exerting effort until they appear to be well-formed and cohesive. Every chronic patient must know that a state of mind is a practical expression of the flow of macro biophysical physiological information flow across neuronal webs connectivity loop transporting information into working memory of the brain to enable reprocessing information and provide an accurate feedback.
All neuronal webs connectivity loops work like electronic networks, indeed brain works to provide the basis for acquiring antistress tools, medium and strategies neutralizing body distresses to predict improvements. By setting up persevering from morning to evening exercises for preventing distress sources, patients must choose only those that are successful exercises, and focus on efforts to create body tranquility that provides pleasant emotions. Everyone in therapy must feel these benefits, because only then will they learn of their own volition, and when the patients take care of their health in their order of priority, they will succeed on the way to recovery.

\section{Fifth, training for assertive interpersonal interactions}

The most satisfying factor for human existence is interpersonal communication in every community. As long as the individual controls the process of relationships with others about their communication in the community, it means that the individual is well balanced (in a physiological homeostatic state) and is well acquainted with the pleasant ways of speaking with others. As long as this situation persists, adapting to changes in interpersonal communication is not an obstacle. When a person loses balance and develops any form of morbid symptoms, there is loss of control over the regulation of sensory and motor systems that interfere with interpersonal communication. This becomes problematic because it is accompanied by either rapid or delayed verbal statements and also in nonverbal expressions such as impulsive or delayed interactions. The most repeatable malign life event scenarios for the overall cluster of subjects suffering from chronic mental (macro biophysical physiological distress) are those that emerge as often the need arises with an anticipated stressful exposure. Therefore, there is no other option rather than gradually training them mapping out all ongoing stress-inducing encounters with whom to precise identify the content and the form of expression (verbal or nonverbal or both in order to literally training to neutralize them.

\section{The rational for training patients for only using assertive skills in social encounters}

The most recurrent scenarios of malignant life expectancy for the general cluster of subjects with chronic mental distress (macro biophysical physiological distress) are those that are often detected as the need arises and are exposed to daily stress intensities that strengthen the distress with the risk of developing further morbidity. Therefore, there is no other possibility, than gradually, to train patients to map all the ongoing encounters that express with whom to accurately identify the content and form of expression (verbal or non-verbal or both) to focus the awareness of the patient's elevation With the therapist and be sure that the instrument will be used verbally and pleasantly without any intention of harming its environment. Therefore, there is no other possibility, than gradually, to train patients to map all the ongoing encounters that express with whom to accurately identify the content and form of expression (verbal or non-verbal or both) to focus the awareness of the patient's elevation With the therapist and be sure that the instrument will be used verbally and pleasantly without any intention of harming its environment. Chronic patients, in particular, need these tools as air because they may first weaken their sensitivity in advance. They will be more confident with how to react and how to react, leaving a place full of stress and no benefit from another negative experience. After training them in the use of a tool that is opposed to environmental pressure, patients will acquire skills for assertive and balance communication that will create a sufficient sense of selfworth and confidence in the trees and will be used in any environment familiar to them. It is known that under such interactions each person 
may acquire an optimal awareness of communication with others under a general set of assumptions: a) be the first to say "peace", b) be very polite, c) always say "thank you" ) Be friendly with others, e) be careful in interaction, $f$ ) feel equal in any social setting, g) do not outsmart his voice and do not yell at anyone, h) do not express any anger or other negative feelings on anyone, l) Do not curse and blacken anyone, $\mathrm{m}$ ) keep secret to yourself for yourself, and so on in a neutral or positive manner.

By any means of such assertive communication, it is possible to preach and prove adherence to a neutral or even positive move. This requires a clinical and home-based training phase and is actually replicated in strenuous social gatherings. In any such assertive media, we can drown ourselves in a neutral or even positive process. It requires a clinical-based training phase and only it actually multiplies in strenuous social gatherings. Let me illustrate examples of this kind of training before exercising in public interactions: 'If you want to understand a subject in a discussion, then just focus your attention on listening to each participant's comments and then follow the subject in the discussion'. If you like to enter a small group of new people, then you must say literally - 'I appreciate the group and want to join you and carefully learn new things you are talking about.

"Do not judge a women for her bad behavior in your eyes, make sure she is not yours'! 'Everyone has the right to express his opinion on any issue without interfering or arguing. 'If you are asked personally, how do you see the subject'? Just say: We share your representation or we have another angle to explain such a problem, now it's our right to bring it without anyone wanting to argue with me. First of all it is essential to digest our point of view and another time to bring it to the discuses. In overall, these maneuverers help in practice lead patients into positive routes of mutual encounters with stress-free interactions.

\section{Six, training patients to deal with personal tasks for problem solving}

There is a general set of assumptions that healthy people use a broad range gained sharp awareness using it in optimal in interactions and problem solving like:

a) Be the first to say 'Hi',

b) Be very polite,

c) Always say 'thank you',

d) Be friendly with others,

e) Be careful in interaction,

f) Feel equal in any social setting,

g) Do not outsmart your voice and do not yell at anyone,

h) Do not express any anger or other negative feelings on anyone,

i) Do not curse and blacken anyone,

j) Keep secret to yourself for yourself, and so on in a neutral or positive manner.

On the contrast, 'mental' patients have to be trained for deep understanding on the nature of approaching no problem solution, by having narrow-based judgements for it during macro biophysical physiological distress. Therefore, they need lasting coaching, in particular, those who need to solve intelligent problems. In order to ensure that they develop a keen awareness of ways to cope with the achievement of practical solutions, they have to know who may assist when a personal problem arises. The specific content of the problem must first be identified. The training requires a clear understanding of the type of problem and is especially related to simple or complex problems. Chronic patients must be well trained to solve simple problems with simple tasks. After the clarity of the problem, must be able to select and assemble at least three tasks in available and easy versions to organize the content and its precise form in successive theoretical steps. The best chosen version must disable the problem in the best possible way. Highly intelligent, highly experienced people who suffer from macro biophysical, physiological distress become limited in the use of their best social skills in their morbid state and act impulsively or in delayed activity.

\section{Rationale for the personal training of mental patients who are required to deal with personal tasks for solving problems}

It appears that chronic mental health care for many years has not been able to crystallize and solidify systematic skills when encountering a recurring problem or a different one. However, most patients have not been able to learn a systematic approach to problem solving. The reason for this is that their voluntary regulation is very limited and personal judgment during the severity is so blocked that in practice there is a loss of new learning ability and voluntary retrieval is blocked and former skills acquired in the past cannot be activated. Under these grounds, with the onset of any remission restoration begins to re-cycle. This still requires the development of very sharp awareness to prevent deterioration! Because it is easier to invest in prevention than to treat the waves of aggravation and reconstruction! The given condition will occur as long as both the therapists and the patients do not know very well that macro biophysical, physiological distresses actually create 'mental disorders' and leave patients without the means and skills to deal with the tasks required in reality. If this is the case, the professionals must have the sharp professional awareness that will be used to explain to the patient his transient condition in order to reduce the disruption of physiological regulation and thereby shorten the deranged power feeding their suffering. As long as there is a distress problem, the voluntary control of self-rule will be very blocked and the illness will continue. So we need to encourage patients immediately, to cooperate in self-regulation into a body and brain calm that will produce pleasant feelings. Under the condition of improving rehabilitation tools will work with the potential for further improvement and over time gradually patients will learn well to differentiate what kind of self-help helps or degrades. In this approach, patients will be taught to transform personal attitudes into concrete tasks, and with them they will be more successful in blocking external and internal stress sources. One may mention, for example, that if people with very high intelligence develop post-traumatic stress, in such a case it would be much better to create a comfortable environment for them, very good sleep conditions, and very regular daily activities. For a while recommend to avoid an exposure to stress sources and receive a sharpening of awareness that they should keep the entire body in a state of tranquility and that as a reward will supply pleasant feelings. Under this condition and later, such people will make an effort to regain their full intelligent control and will be extremely aware how and why they need to maintain the overall organism in a calm state.

\section{Seventh, setting up family and social stress-free constellation of antecedent conditions (COAC)}

Mental health education focuses on creating 'support' and 'protected social environments'. However, by replacing the concept 
of "mental health" with the redesigning of the body, brain and mind unity around the clock, the body's operating range (BOR) in the daytime awake at nighttime sleep, the intelligent self-navigation is basically guiding the focus on tools, skills and tasks that restore normal navigation and the creation of a macro biophysical physiological resilience to recover and maintain balance activity. All the tools, skills and tasks together and separately are based on basic principles that shape the stress-free internal systems and systems in interpersonal relationships, also without stress and conserved equilibrium. To achieve this goal, a neuro-psychotherapist must be well aware of the essential need to provide assistance to any mental patient and to set for him only actualized COAC's in theoretical design and, of course, to act as a barrier to neutralizing stress exposure. A stress-free positive COAC release patients from heavy daily burdens and provide favorable conditions for arranging one's good quality of life.

\section{Rationale for setting up family and social stress-free constellation of antecedent conditions (COAC)}

Psychiatric patients who are overly sensitive to normal stress also suffer from a lack of strategies, means, techniques, and personal skills how to self-schedule the COAC.

It is perfectly understandable that any kind of "immediate environment" that includes family members, friends, colleagues and local communities does not have any insight into building interpersonal encounters on the basis of COAC's stress-free meetings. For this reason, it makes sense that neuro-psychotherapists will be very conscious in order to encourage patients to agree on the need to invite the most appropriate person to fit positively with whom she/he is in contact to begin building the COAC.

Two basic principles shape any positive COAC. The first is to provide a high awareness among patients not to talk to people around them about their own negative feelings or situation. Not to preoccupy himself with thoughts of 'self-shame,' 'personal guilt', or whatever negative thoughts about himself. Why? The first principle is because of self-reflection as evil by intrusive thoughts that shift the macrobiophysical physiological into permanent distress! The second is constant awareness on maintaining the need to anticipate any known stressor in order to prevent it from the formation of a lasting macro biophysical physiological distress! In other words,

neuro-psychotherapists must be duty-bound at these critical points. Guidelines should monitor 'mental' patients and lead them from pathology to recovery and relapse prevention.

\section{Clinical Case Vignettes: General Principles Attributed to Case Management}

All clinical cases use the same neuro-psychotherapeutic principles based on:

1. Presenting essential guidance with theoretical baselines on normal body-brain-mind macro biophysical physiological neuropsychological operational mechanisms automatically operating under one's intelligent level, preserving health, wellbeing and quality of life.

2. Applying to any patient the same slogan: there is no replacement for life, there is no replacement for health, and there is no replacement for personal quality of life! It helps in cementing directions for realizing experts guidance from abnormal into normal states.

3. Explaining in understandable language, healthy people are resilient for active sustaining their inner balance (homeostasis) of macro biophysical physiological body operational ranges (BOR) with four driven automatic nighttime sleep stages and four wakeful (conscious) BOR stages, with High-tech like properties (living biological machinery).

4. Entraining basic knowledge of people who lost their health and feel discomfort with other bother symptoms to be in transitory homeostatic deregulating (THD) condition, that is treatable into transitory homeostatic resynchronizing (THR) healthy remission.

5. Bringing knowledge to people with THD who are highly susceptible to personal stress that induces macro biophysical physiological distress from which they suffer.

6. Mapping the personal relevant operating stress-inducing factors originating macro biophysical and micro biochemical physiological signs and symptoms to block them.

7. Stress neutralizes the effect of drugs, rather than neuropsychotherapy. The advantage of neutralization is enhanced and helps in training patients how and why distress must be blocked in the first place!

8. Helping patients to identify self-experienced macro biophysical physiological distress measured with BOR scores between 1 at rest and 4 at maximal load intensity.

9. Entraining readiness for compliance with "here and now" based on real life stress-inducing factors helping in reframing life event scenarios based on stress-free encounters.

10. Using systematic active tasks to build family and social positive COACs to protect patients' from family discourse, social troubles with clear awareness about their favorable effects.

11. Consistently blocking off intrusive symptoms and thoughts with active shifting one's attention focus on tranquil or neutral sources located across external world.

12. Protecting self from clinical symptoms discussion with nonmedical experts and avoiding passive or active topics about 'treatment' approaches.

13. Protecting self by teaching her/him acquiring skills for assertive interactions in encounters with family members, friends and local community.

14. Acquiring personalize strategies and tools for concrete problemsolving techniques.

15. Sharpen awareness for refining one's self-monitor sustenance of balanced sleep, nutrition, work, creativity and rest under tranquility.

16. Sharpen patients' awareness for daily resynchronizing THD with physical exercises, running or walking to raise personal capacity to reach body tranquility and peace of biophysical mind as expressions of temporary THR.

17. Sharpen neuro-psychotherapists awareness for possessing valid capacity and competence in using tools and skills helping her/him to adapt to any predictable daily life scenario and be able to balance selfregulation.

\section{Short Presentation of Case I}

Male patient, age of 70 arrived with complaints having troubles falling asleep, nightmares, lack of energy, tiredness, and lack of 
concentration, intrusive thoughts, anxieties, gloomy mood, and loss of interest for social contacts. He stated that he went through a thorough clinical examination, including neurological and that all medical experts declared were 'organically healthy but having only psychological problems'! Even one neurologist put it 'everything is in your mind'! Perhaps the last statement was a cornerstone driving him to contact me for hearing from some therapist that in our practice were less drugs and more training for a better quality of life. During first interview, we identified many, but mapped out his most troubled daily stress-inducing factors. We instantly said to him: "You're a strong man suffering so long! We wouldn't be able so long searching for help." Afterwards, We presented him with two diagrams on how general physiology and 'neurophysiology', in particular, works in synchrony in health and otherwise in diseases. Moreover, "I told him it's not your fault! It is a medical problem that were missed out for lack of knowledge. Present Medicine and Psychology try to correct such states like your' transitory homeostatic deregulating (THD) state. We initiated training him on spot of how and why it is necessary each day to block up intrusive thoughts by shifting attention on neutral things, doing exercises, making walks due to his physical ability using his inner feelings as an indicator that he does it suited for right. He just wanted 'getting out of his symptoms' and 'live his life in his way'. 'By now you have THD relapse and help me please to help you'. 'I gave him a list of recommendations keeping him busy to divert his intention from the body to the outside of him. We asked him how and why he should learn self-control over driving his 'body operational ranges (BOR) to gain 'pleasant biophysical physiological emotions':

1) Keep on with anti-depression drug you take and tell your family physician that to avoid side effects you'll be better on moderateminimal dosages and add neuropsychological tools like below:

2) Avoid old and new stress-inducing events to, a priori, counteract existing physiological distress.

3) Keep a daily active life schedule.

4) Keep a balanced sleep by avoiding watching TV and radio transmissions upsetting him with topics after about 19.00 P.M. and engage himself afterwards with neutral or enjoying neutral states like moderate walking, games, readings, movies and so on.

6) Avoiding any discussion on personal problem with anybody in order to prevent deterioration of symptoms.

7) Avoid self-upsetting and annoying topics discus only with me or your family doctor.

8) Block up intrusive thoughts by paying attention to personalize events occurring out of the body by shifting attention to any neutral or positive charged event in a systematic way. We added, 'your pathophysiology automatically pulls you into your open working memory centers constantly supplying you with garbage-like information'. In following sessions we add explaining how to close up some memory repositories that are open and bad thoughts that bother': a) persistently train to keep yourself in focus on point seven $(7+8)$, on the list. And b) find and entrain an event that optimizes your instant shifting attention focus to continue be able catch your on some favorable relaxing object or subject or environmental scene to create in your body calmness with good feelings.

9) Pacify yourself by talking in silence to self about 'I'm in the rechangeable state and I'm sure I'll go through it'.
10) Occupy self with self-awareness 'on identifying social or other causes worsening intensity of signs or emergence of new symptoms.

11) Avoid listening to other 'sick subjects complaints and means of remedy' that may increase your morbid hesitation to follow given instructions.

12) Avoid actively ruminate about past and future unpleasant outcomes, since they bring in worsening of symptoms and bad feelings.

We had biweekly therapeutic sessions lasting about 30 minutes each. Grade intensity weakened from point 4 with maximal to point 1 resting symptom-free by about six weeks after onset of our treatment. He kept on sessions for about another 6 months once in a month stopped taken medicine and further visited from time-to-time his family physician for real organic reasons.

In case of him there were no needs to invite his family members to train them too in this way, to create a family support system. Reciprocally, in cases of need troubled family members were been invited too. Its design for reframing family's constellation of antecedent conditions (COAC) under stress into COAC with stress-free daily coexistence.

\section{Case II}

Married female, housekeeper of 32 years old, lives with her husband of 38 years old and three kids. Her husband is a self-employed engineer who has financial troubles handling his business and she helps him to overcome difficulties, but this puts both into fruitless debates. Her mother-in-law keeps interfering and supports her son's in such debates, infuriating her badly. She went through longlasting medical check-ups that were 'organic-free'. She arrived with her husband presenting following symptoms: typical shortness of breath, accelerated heartbeats, nausea, phobias making visits to her mother-in-law impossible. She had fears taking a flight that increased nightmares during nighttime superficial sleep. She felt depressed, tired and exhausted, having lost her power to debate with her husband on his business. After mapping out her main stress-factors, we introduced them to our approach in perceiving macro biophysical physiological neuropsychiatric operational mechanisms to restore her upset biophysical physiology. Straightforward our strategy was : 'Instead of you efficiently controlling your body, you became a slave to your body! 'In fact, you got a transitory homeostatic deregulated (THD) condition and it is fully treatable and manageable in two-phases'. We continued: 'First, you should acquire tools and means on why you need to learn how to overcome your symptoms. Second, you will continue gaining needed strategies and techniques helping to protect your physiology and raising your physiological resilience to allow you genes executing its genetic control during your nighttime sleep circadian cycles'.

Soon after, her husband suddenly said he thinks his physiology is out of order too. 'I am nervous, have many wakes and we always fight with my legs during sleep'- he finished. We told him to go to his family physician for medical checks, prior to taking any essential steps in neuro-psychotherapy. 'However, you may listen carefully to our recommendations for your wife and fit some of our instructions to your needs too'. Complementary We noted: 'deregulated physiology has to be replaced with normal regulation achieved with many means'. Like in case 1 , We recommended her to:

1) Use the best assertive strategy with her husband in his presence: 'You have an equal right to your opinion with the same equal duty to respect his right on his opinion!' 'It means that both have the same 
right and duty as a couple. Our strategy here was: 'You both must learn essentials of accurate listening to each other's meaning without disruption, only to comprehend the whole message'. That presented for both a learning opportunity; precisely identifying each other's accurate intention. 'Do not hurry to give an instant answer'. Take your time to 'sleep it over' separately at breaks to avoid forcible solutions on spot. Think about flexible alternatives and in case you will find none bring it into our next session'.

2. 'Do not discuss financial business problems with your husband to block off their stressful nature'.

3. We instantly encouraged her husband to take advice from a financial expert and he agreed.

4. 'Tell your children that you have a transitory physiological (medical) condition easy treatable to calm them down and helping you to carry on your duties in a personal pace'. The rational for it is that all of you must be at home under tranquil states.5. 'Both of you should be fully aware avoiding after 19.00 P.M. any mutual misunderstanding and exposure to unpleasant social encounters'. The rational for it is to prevent stress-inducing acts.

6. 'Both of you should at all times create calming conditions before going to sleep.

7. The strategy: if in case you get a panic attack awakening from sleep count aloud for self ' 1 inhale and 2 exhale and repeat it in a slow tempo several times and /or take an anti-anxiety prescribed medication and to calm down to return to sleep

8. 'Before entering the bed tell yourself silently 'there is no lifethreatening situation and We need sleeping until morning hours'. The rational for it is that it has a favorable medical effect weakening the bonds in the pathological neuronal webs connectivity loop that is behind her panic attack symptoms.

9. 'You should be aware of, for next four weeks, avoiding meetings with your mother-in-law'. The rational for it is protecting both of you from further interactional deterioration.

10. 'You should teach yourself to block off self-inducing repetitions about your symptoms and stressful social encounters'.

The rational for it focuses on point eight (8). After a month, her symptoms gradually weakened. At this time, her husband went through an entire set of medical checks being 'organic-free'. We reinforced both with recommending them to keep their physiology under selfcontrol with two fundamental but inseparable parameters: a) keeping tranquility around the clock, b) keeping body operational ranges (BOR) under minimal-moderate loadings with positive emotional experiences.

After two months, We invited her mother-in-law with the couple. We designed such strategy to provide positive connotations to mother-in-law intentions by backing her son. In their presence, We told the mother-in-law that her son and daughter-in-law have been physiological oversensitive subjects that respect very much and appreciate her help in raising their children. We explained to her, in short about macro biophysical physiological disturbances being in the origin of biophysical physiological (mental) disorders. We asked her to keep along with her children and grandchildren interactions free of debates. She willingly accepted our instructions. We kept sessions once a month for about one year. During that interval both acquired full knowledge on how to protect themselves from personalize stress- inducing factors. She stopped advising her husband on business budget. He took a financial adviser. Assertive interaction she performed in encounters with her and mother-in-law. They kept on been further in contact only with her family physician.

Both cases were under accurate guidance in line with macro biophysical physiological neuropsychiatric operational mechanisms, which replace deregulation with normal regulation in their mutual daily living conditions. One must recall the social biased truth, that it is easy to de-stigmatize and accept physiological disturbance rather than the 'stigma of mental'. Nevertheless, it is useful to know that in both cases, like in many others that these subjects at risk eventually comprehend the naked truth about the detrimental effects inflicted by transitory biophysical-biochemical physiological disturbances. More importantly, were the essential facts that they regained self-control over their stress-free daily life framework.

Further Macro Biophysical Physiological Neuropsychiatric Progress. Neuropsychiatry (neuropsychology) bases and orients macro biophysical physiological operational mechanisms on initiating, reframing and leading stress-free external COAC, actively helping replacing THD relapses with THR remission levels. However, as a practice it has an essential definite drawback and only in subjective implementation. The disadvantage stems from the absence of so far tests obtained using an electronic device measuring the intensity of symptoms at relapses and THR conditions after their removal. Such accurate objective measurements are in need for three reasons:

1. Arriving to an inter-correlated objective and subjective measurements providing a unifying coefficient for defining personalize level of THR remission.

2. Defining personalize level of a unifying coefficient determined by the range between lower-to-upper thresholds of homeostatic frames of reference.

3. Training patients using self-control for sustaining a unifying coefficient within an optimized level. On the basics of above, it is clear that neuropsychiatrists in practice and research need a mobile macro biophysical analyzer (MBA), acting like diagnostic brain imaging techniques, but with cheap non-invasive measurements. For this to occur, in our opinion there should be a micro/macro biophysical physiological neuropsychiatric center for practice and research. The latter should advance compatible standardize neuropsychiatric scales and an assembled computer software on available strategies and tools for developing practical instrumental skills for personalize usage. Neuropsychotherapy based on macro biophysical physiological operational mechanisms is able initiating, reframing and leading stress-free external (COAC) actively helping replacing THD relapses with THR remission levels.

\section{Conclusion}

Neuropsychotherapy is suing a term belonging primarily to a medical profession. As such, it straightforwardly treats etiological sources, pathogenesis, clinical manifestations, prognosis and prevention. The model is the same as with any other medical model. It feeds all the benefits that are in usage by traditional psychotherapy. Nevertheless, the great difference is that psychotherapy is not standing on theoretical grounds of macro biophysical physiological neuropsychotherapy. Neuropsychotherapy neither challenges nor rejects any of psychotherapeutic, traditional Oriental, alternative or complementary methods. In fact, all of their information units base rely on biophysical physiological information units in nature. 
Citation: Yakov N (2018) Macro Biophysical Physiological Neuropsychotherapy: Theory and Practice. J Ment Disord Treat 4: 156. doi:10.4172/2471271X.1000156

Page 12 of 12

Therefore, just those procedures bearing ranquil physiologicalinducing responses, possess strong attachment to 'here and now' dynamics between therapist and patients interactions. Its method precisely employs macro biophysical physiological neuro-operational mechanisms imbedded in given strategies, means and tools navigating the process of substituting transitory homeostatic deregulation (THD) relapses with transitory homeostatic resynchronizing (THR) remission levels keeping in long-run. Complementary, it advocates usage of all other known to medicine interventions improving physiological health and wellbeing. This method anticipates the introduction of an electronic equipment for measuring non-invasively macro biophysical physiological parameters in order to improve its diagnostic, prophylaxis and treatment efficacy.

Neuro-psychotherapy is a unique medico-psychological method that presents and describes its theoretical, macro biophysical physiological base, in which its practice is based on valuable tools and its significance works on the macro physiological levels in order to reshape the tiny mechanisms of deregulations in the body, brain, and mind in patients with 'Mental disorders'. 'Unique neuropsychological strategies, tools, and skills self-guide the macro biophysical physiological processes until the synchronic biomarkers appear to sharpen awareness, which soothe the whole body that produces pleasant internal emotions in the limbic brain. The two parameters, overall physical calmness and pleasant feelings indicate evidence that they have replaced transitory homeostatic deregulation (THD-'mental disorder') with a transitory homeostatic resynchronizing (THR) remission. As long as the THR activity for many years across the unified body, brain and mind, is well maintained every day, it helps to weaken the influence of abnormal genes that allow patients to enjoy a healthy lifestyle. Clinical evidence suggests that THD cases with self-regulating efforts have been able to influence abnormal genes and stress-ridden environments, thus removing the latter and improving THR for many years in health and function. These strong evidences cannot be undone because neuro-psychotherapy experts have been given a good longterm opportunity to rehabilitate the stress-induced environment while simultaneously attenuating abnormal genes to zero effect, proving that they have undergone a new normal mutation. This intelligent approach maintains a lifespan in the self-regulation of healthy, productive macro biophysical physiological operations, which ensures constant creativity and a good quality of life.

\section{References}

1. Thombs BD, de Jonge $\mathrm{P}$, Coyne JC, Whooley MA, Frasure-Smith $\mathrm{N}$, et al (2008) Depression screening and patient outcomes in cardiovascular care: a systematic review. JAMA 300: 2161-2171.
2. Monnikes H, Tebbe JJ, Hildebrandt M, Arck P, Osmanoglou E, et al. (2001) Role of Stress in Functional Gastrointestinal Disorders. Evidence for StressInduced Alterations in Gastrointestinal Motility and Sensitivity. Dig Dis 19: 201-211.

3. Keyes KM, Hatzenbuehler ML, Grant BF, Hasin D (2012) Stress and Alcohol. Epidemiologic Evidence. Alcohol Res 34: 391-400.

4. Lockwood PL, lannetti GD and Haggard P (2013) Transcranial magnetic stimulation over human secondary somatosensory cortex disrupts perception of pain intensity. Cortex. 49: 2201-2209

5. Naisberg Y (2015) Macro Biophysical Physiological Neuropsychiatry. Brain Disord Ther 4: 157

6. Naisberg Y (2015) Biophysical Mind Units and Leibniz's Law in Practice. J Psychiatry 18: 294.

7. Naisberg Y (2015) Biophysical Mind-Brain Sleep Regulation in Health and Pathology. J Neurol Neurobiol.

8. Leão AAP (1944) Spreading depression of activity in the cerebral cortex. J Neurophysiol 7: 359-390.

9. Herreras O, Largo C, Ibarz JM, Somjen GG, Martin del Rio R (1994) Role of neuronal synchronizing mechanisms in the propagation of spreading depression in the in vivo hippocampus. J Neurosci 14: 7087-7098.

10. Martins-Ferreira H, Nedergaard M, Nicholson C (2000) Perspectives on spreading depression. Brain Res Brain Res Rev 32: 215-234.

11. Hadjikhani N, Sanchez del Rio M, Wu O, Schwartz D, Bakker D, et al. (2001) Mechanisms of migraine aura revealed by functional MRI in human visual cortex. Proc Natl Acad Sci USA 98: 4687-4692.

12. Chuquet J, Hollender L, Nimchinsky EA (2007) High-resolution in vivo imaging of the neurovascular unit during spreading depression. J Neurosci 27: 40364044.

13. Lauritzen M, Dreier JP, Fabricius M, Hartings JA, Graf R, et al. (2011) Clinical relevance of cortical spreading depression in neurological disorders: migraine malignant stroke, subarachnoid and intracranial hemorrhage, and traumatic brain injury. J Cereb Blood Flow metab. 31: 17-35.

14. Dreier JP (2011) The role of spreading depression, spreading depolarization and spreading ischemia in neurological disease. Nat Med. 17: 439-447.

15. Aiba I, Shuttleworth CW (2012) Sustained NMDA receptor activation by spreading depolarizations can initiate excitotoxic injury in metabolically compromised neurons. J Physiol 590: 5877-5893.

16. Pietrobon D and Moskowitz MA (2014) Chaos and commotion in the wake of cortical spreading depression and spreading depolarizations. Nat Rev Neurosc 15: 379-393.

17. Dux PE, IvanoffJ, Asplund CL, Marois R (2006) Isolation of a Central Bottleneck of Information Processing with time-resolved fMRI. Neuron 52: 1109-1120.

18. Kim SY, Kim MS, Chun MM (2005) Concurrent working memory load can reduce distraction. Proc Natl Acad Sci U S A 102: 16524-16529.

19. Hesselmann G, Flandin G, Dehaene S (2011) Probing the cortical network underlying the psychological refractory period: A combined EEG-fMRI study Neuroimage 56: 1608-1621. 